\title{
A STUDY OF SPATIO-TEMPORAL SPREAD OF INFECTIOUS DISEASE: SARS
}

\author{
AFIA NAHEED \\ (Received 12 January 2016; first published online 26 September 2016)
}

2010 Mathematics subject classification: primary 92D30; secondary 65C20.

Keywords and phrases: SARS (severe acute respiratory syndrome), mathematical modelling, infectious diseases, parameter estimation, sensitivity and uncertainty analysis.

This thesis is based on using three different types of deterministic compartmental epidemic models to investigate the transmission dynamics of severe acute respiratory syndrome (SARS). These models are represented by ordinary and partial differential equations, with the inclusion of a reaction-diffusion system. The first model assumes susceptible-exposed-infected-diagnosed-recovered (SEIJR) populations, whereas the second and third models are extensions of the first model, with treatment and quarantine compartments added. For different initial population distributions the system of differential equations, representing different compartments, has been solved in the presence of diffusion in the first three compartments (that is, susceptible, exposed and infected). In this study the effects of diffusion on SARS transmission are investigated as are the effects of some intervention strategies. It is shown that diffusion and initial population distribution play crucial roles in disease transmission. Then, the same system is solved numerically with diffusion in the susceptible, exposed and infected compartments and with cross-diffusion in the susceptible and exposed compartments for different cases. Using clinical and demographic information for SARS, the SEIJR model is further extended to a susceptible-exposed-infecteddiagnosed-treated-recovered (SEIJTR) model. The SEIJTR model's parameters are estimated, again using available field data on the 2003 SARS epidemic in Hong Kong. After that, model parameters are analysed for sensitivity and uncertainty. Three different techniques are used to perform the sensitivity analysis. The effect of the treatment compartment on SARS transmission is then numerically studied. Stability analyses of steady-state and treatment-reduced basic reproduction numbers are performed. Studies show that availability of treatment can reduce infection

Thesis submitted to Swinburne University of Technology in February 2015; degree awarded on 31 July 2015; supervisors Manmohan Singh, David Lucy and David Richards.

(c) 2016 Australian Mathematical Publishing Association Inc. 0004-9727/2016 \$16.00 
significantly. Finally, a quarantine compartment is added to the SEIJTR model in order to study the effects of quarantine on SARS transmission. The results for this extended SEQIJTR (adding Q for quarantine to SEIJTR) model are compared with those for the SEIJTR model in which only isolation and treatment but no quarantine measures are used as intervention. The investigations show that the presence of quarantine measures effectively reduces disease transmission.

AFIA NAHEED, Mathematics Discipline,

Faculty of Engineering Science and Technology,

Swinburne University of Technology,

Hawthorn, Victoria 3122, Australia

e-mail: afia_naheed@yahoo.com 